\title{
Methods for cleaning turbid nematode suspensions collected from different land-use types and soil types
}

\author{
Jie Zhao ${ }^{1,2}$, Kelin Wang ${ }^{1,2, *}$ \\ 1 Key Laboratory of Agro-ecological Processes in Subtropical Region, Institute of Subtropical Agriculture, Chinese Academy of Sciences, \\ Changsha 410125, China \\ 2 Huanjiang Observation and Research Station for Karst Ecosystems, Chinese Academy of Sciences, Huanjiang 547100, China
}

H I G H L I G H T S

- Soil nematode samples can be quite turbid, which are not satisfactory for microscopy.

- Three methods were designed for cleaning turbid nematode suspensions.

- Nematode abundance did not significantly differ among control and the three methods.

- Repeated centrifugation had slightly higher recovery rate of nematodes than the other methods.

\section{ARTICLE INFO}

Article history:

Received April 22, 2021

Revised June 12, 2021

Accepted July 9, 2021

Keywords:

Soil nematodes

Purification method

Centrifugation

Low-temperature settlement

Luvisols

Acrisols
GRAPHICAL ABSTRACT

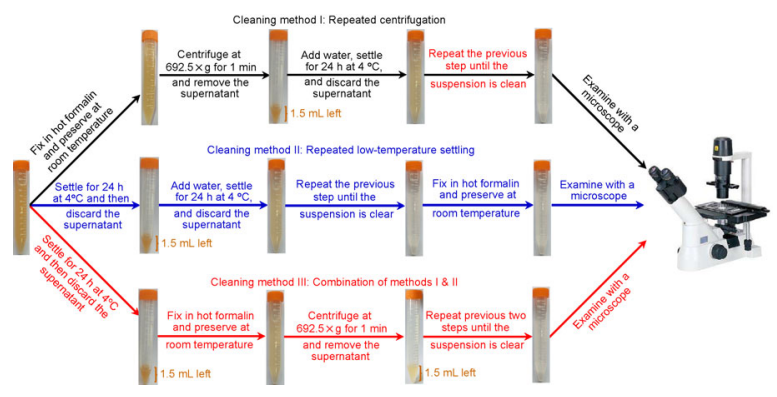

A B S TR A C T

Soil nematodes are useful ecological indicators and can be extracted from soil by a variety of techniques. Because the extracted nematode samples (suspensions) can be quite turbid (i.e., they contain soil particles and organic particles in addition to nematodes), quantitative and taxonomic analyses of the nematodes by microscopy can be difficult. In this study, the following three methods for cleaning turbid suspensions obtained from Baermann funnels were assessed: repeated centrifugation at $692.5 \times g$ for $1 \mathrm{~min}$, repeated settling at low-temperature $\left(4^{\circ} \mathrm{C}\right)$ for $24 \mathrm{~h}$, and a combination of low-temperature settling and centrifugation. Nematodes were extracted with Baermann funnels from soil samples collected from four land-use types (since land-use type can affect the turbidity of nematode suspensions), and the resulting suspensions were cleaned by the three methods before nematode abundance was assessed. As a control, samples (i.e., suspensions) were simply diluted with water, and nematodes were counted in the entire volume. The results showed that, within each land-use type, nematode abundance did not significantly differ between the control and the three cleaning methods. Averaged across all land-use types, however, the nematode recovery rate was slightly higher with repeated centrifugation than with the other two cleaning methods. Therefore, the proposed methods are sound for cleaning turbid nematode suspensions, and repeated centrifugation is the most efficient method.

(c) Higher Education Press 2021
* Corresponding author

E-mail address: kelin@isa.ac.cn (K. Wang)

\section{Introduction}

Soil nematodes are excellent bioindicators of ecological status and have been widely used for environmental monitoring (Bongers and Ferris, 1999; Zhang et al., 2019; Neher, 2001; Aira and Domínguez, 2020; Chen et al., 2020). The use 
of soil nematodes as bioindicators requires their extraction from soil, which can be conducted with a variety of techniques. Because nematodes live in the water films around soil particles, most nematode extraction methods, whether active or passive, use water as an extracting agent. The methods are primarily based on the nematode movement, nematode density, and/or body size (Coleman and Crossley, 1996; Coleman and Wall, 2015). The current study concerns modifications of the Baermann funnel method and the sieving and sucrose-centrifugation method with the goal of obtaining cleaner (less turbid) nematode samples.

In the standard Baermann funnel method, nematode movement and nematode density relative to water cause the nematodes to move downward out of the water-saturated soil sample, through a filter, and into the stem of a funnel, where the nematodes can be collected in a suspension (Barker et al., 1985; Coleman and Crossley, 1996; Zhang et al., 2013). This method can mainly extract alive nematodes. The soil nematodes with poor mobility (e.g., dead or nearly dead nematodes and sedentary endoparasites) generally cannot be extracted by the Baermann funnel method. The sieving and sucrose-centrifugation method has higher efficiency in extracting soil nematodes (including dead or nearly dead nematodes) than the Baermann funnel method. In the standard sieving and sucrose-centrifugation method, nematodes are first separated from denser soil particles by mixing the soil sample in water, allowing the denser soil particles to settle while the nematodes are still in suspension, and then collecting the nematodes (plus less dense soil particles and organic particles) by washing the suspension through a sieve of an appropriate mesh size. The collected nematodes are then added to water, and the suspension is centrifuged such that the non-nematode organic matter floats while the nematodes and soil particles form a pellet. The liquid with most of the non-nematode organic matter is discarded, and the pellet is suspended in a sucrose solution of an appropriate density. The solution (i.e., suspension) is then centrifuged such that the nematodes tend to float in the liquid whereas the soil particles tend to form a new pellet. The liquid containing nematodes is poured through a sieve of an appropriate mesh size, and the nematodes are washed from the sieve into a vial (Freckman and Baldwin, 1990; Coleman et al., 1999). For both extraction methods, the vials containing the extracted nematodes are left undisturbed in water for at least $2 \mathrm{~h}$, during which time the nematodes settle but most remaining organic particles do not. The organic particles can then be discarded by carefully removing most of the water in the vial without disturbing the nematodes at the bottom (Wright and Coleman, 2000; Sarathchandra et al., 2001; Geisen et al., 2018).

Regardless of the extraction method, the vials of nematode suspension may contain many mineral soil particles and organic particles in addition to nematodes, i.e., the suspensions may be turbid (Supporting information, Fig. S1). This is especially true for soils with a high clay content (Seinhorst, 1962). Such turbid suspensions make the quantification and identification of nematodes via microscopy very difficult
(Supporting information, Figs. S1 and S2). In our previous research (Zhao et al., 2012; Zhao et al., 2015; Li et al., 2020), we found that the turbidity of nematode suspensions obtained from Baermann funnels was affected by land-use type (unpublished observations). In the current study, we assessed three methods for cleaning nematode suspensions obtained from Baermann funnels. The soil samples included both red soil and the calcareous soil and were collected from four landuse types (cropland, natural grass-shrubland, orange orchard, and pine forest).

\section{Materials and methods}

The soil samples in this study were collected in Huanjiang County, Guangxi Province, China, where calcareous soil and red soil are the typical soil types (Xiao et al., 2014; Huang et al., 2018). Areas with the following land-use types were sampled: cropland, grass-shrubland, orange orchard, and pine forest. The cropland and the grass-shrubland have typical Luvisols (i.e., calcareous soils) (FAO, 2006) and the orange orchard and the pine forest have typical Acrisols (i.e., red soils) (FAO, 2006). The nematode suspensions obtained via Baermann funnel extraction of the soil samples (an Acrisol) from the orange orchard were very turbid (Supporting information, Figs. S1 and S2) and could not be directly examined by microscopy for taxonomic or quantitative determination of nematodes.

In March 2019, four replicate sites were selected for each of the land-use types, and one $30 \mathrm{~m} \times 30 \mathrm{~m}$ plot was established at each of the 16 sites. After the surface litter was removed, soil cores (5 cm in diameter) were taken at 0-10 cm depth from 25 random locations in each plot. The 25 cores from each plot were combined to form one composite sample, yielding four replicate samples for each land-use type. Samples were transported to the laboratory in insulated boxes. The soil was then passed through a 2-mm sieve to remove roots and small stones. Nematodes were extracted from $100 \mathrm{~g}$ of moist soil using the Baermann funnel method (Barker et al., 1985); the soil was placed directly on the funnel without prior decanting and sieving. Four 100-g subsamples were extracted from each soil sample and were subjected to the cleaning methods described in the following paragraphs.

Three cleaning methods were assessed: repeated centrifugation; repeated natural settling at low temperature $\left(4^{\circ} \mathrm{C}\right)$, and a combination of low-temperature settling and centrifugation (Fig. 1). Low temperature can reduce nematode degradation during settling. The centrifugation method (method I) involves centrifuging the suspension (first in formalin and then in water), discarding of the supernatant, and adding water; these steps are repeated until the sample appears to be clean. The nematodes in the final water suspension are examined with an inverted microscope (Eclipse Ts100, Nikon). The low-temperature settling method (method II) involves settling in water at $4^{\circ} \mathrm{C}$, discarding of the supernatant, and adding water; these steps are repeated until 


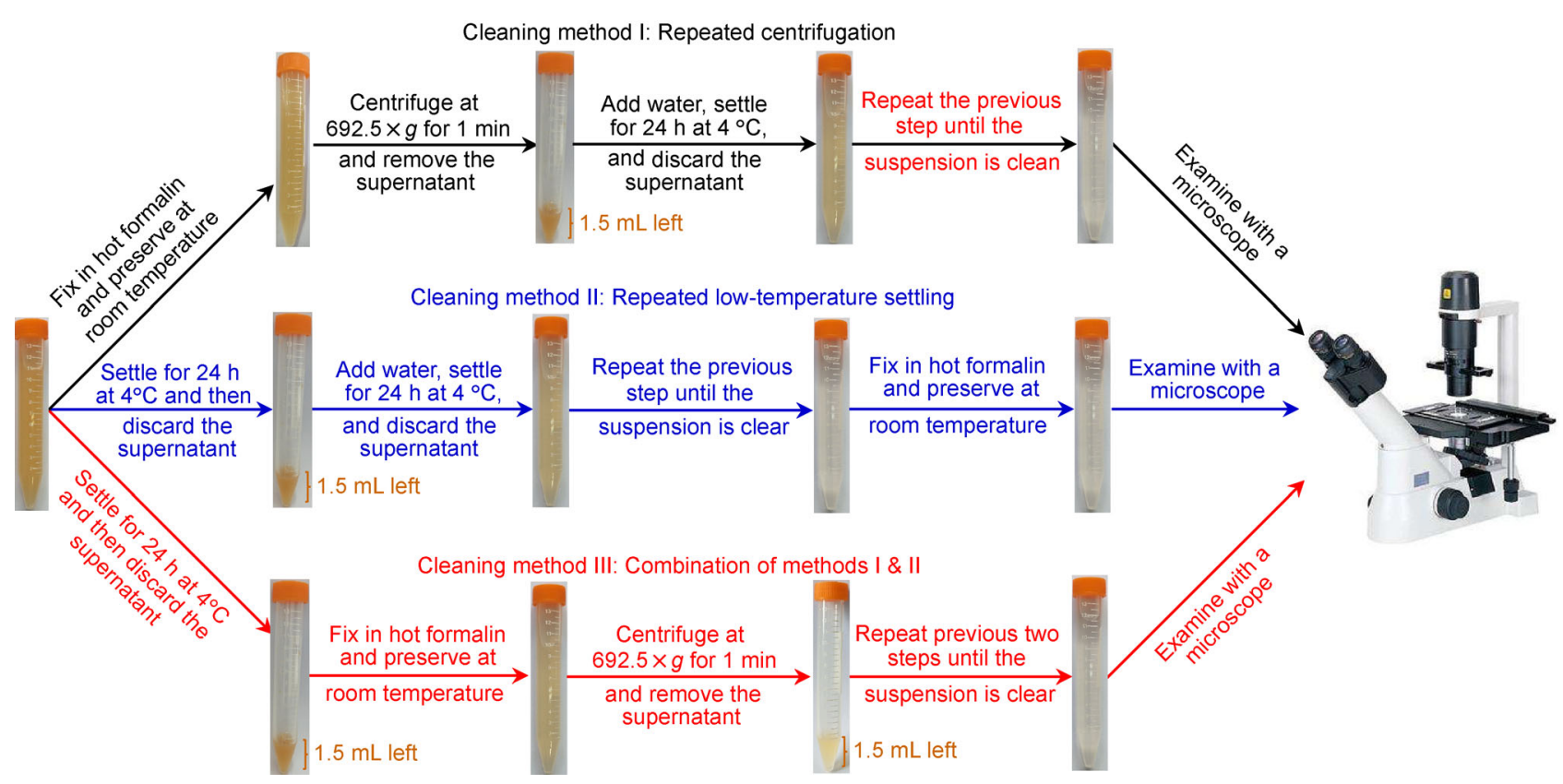

Fig. 1 Flow chart of the three methods that were compared for cleaning of turbid nematode samples.

the sample appears to be clean. The nematodes are then preserved in formalin before they are examined with an inverted microscope. The combined low-temperature settling and centrifugation method (method III) involves settling in water at $4^{\circ} \mathrm{C}$, discarding of the supernatant, and fixation in hot formalin, followed by the repeated centrifugation in hot formalin and discarding of the supernatant until the sample appears to be clean. The nematodes in formalin are then examined with an inverted microscope. For all steps and all methods, the samples were in 15-ml centrifuge tubes $(\sim 12 \mathrm{~cm}$ in height). In this investigation, regarding the centrifugation procedure, a low-speed desktop centrifuge (Anting TDL-40B, China) was used.

A preliminary experiment was conducted to determine the centrifugation speed to be used in methods I and III, using soil samples from grass-shrublands. The centrifugation speed should be sufficient to concentrate the nematodes in a pellet (the recovery rate should be near $100 \%$ ) while leaving as much other material as possible in the supernatant. Nematode suspensions in water were obtained directly from Baermann funnels. We evaluated the recovery rate of nematodes at seven centrifugation speeds: 4000, 3000, 2500, 2000, 1500, 1000 , and $500 \mathrm{rpm}$ which corresponding to $2770.0,1558.1$, $1082.0,692.5,389.5,173.1$, and $43.3 \times g$. To reduce the time required for processing samples in methods I and III, the centrifugation time was set at $1 \mathrm{~min}$ rather than the commonly used 3-5 min. The results showed that the nematode recovery rate did not significantly differ with centrifugation speeds of $2770.0,1558.1,1082.0,692.5 \times g$ and that the recovery rates were significantly higher at these speeds than at $389.5,173.1$, and $43.3 \times g$ (Fig. 2). We therefore selected $692.5 \times g$ as the centrifugation speed for methods I and III. For methods II and III, we allowed nematodes to settle at $4^{\circ} \mathrm{C}$ for $24 \mathrm{~h}$ based on

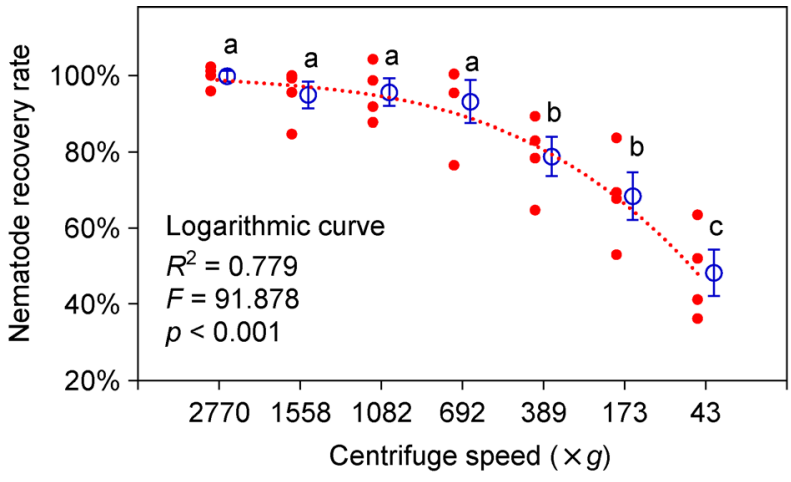

Fig. 2 Relationships between nematode recovery rates (\%) and centrifuge speeds. Nematode samples were centrifuged for $1 \mathrm{~min}$ at $2770.0,1558.1,1082.0,692.5,389.5,173.1$, and 43.3 $\mathrm{xg}$. The recovery rate of nematodes was the number of nematodes counted after centrifugation divided by the number counted before centrifugation $\times 100$. Each dot represents one replicate $(n=4)$. The curve is the fitted regression curve. Circles and error bars indicate means \pm SE. Means with different letters are significantly different at $p<0.05$.

previous reports (Wright and Coleman, 2000; Geisen et al., 2018).

There were other differences among the cleaning methods (Fig. 1). In method I, samples were fixed in hot formalin before purification because the soil samples were extracted at a field station where a centrifuge was not available. Method II was designed to clean fresh nematode samples, which were then fixed in hot formalin so that microscopic examination could be delayed. Method III first used low temperature settling and then fixation in hot formalin; the samples were further cleaned by repeated centrifugation. In method III, the initial settling was 
not repeated because doing so would take several days, during which time nematodes might degrade.

A control method was also used. For the control, the suspensions from the Baermann funnels were diluted with water until the turbidity was greatly reduced. The suspensions, which consequently had greatly increased in volume, were then examined. We assumed the nematode recovery rate with the control was $100 \%$. As noted above, four subsamples were extracted via Baermann funnel from each soil sample. Each of the four resulting nematode suspensions was then randomly assigned to the control or to method I, II, or III.

One-way ANOVAs were used to determine the effects of land-use type on the turbidity of the nematode suspensions before cleaning (Supporting information, Fig. S2) and the effects of cleaning method on nematode abundance and recovery rate (Supporting information, Fig. S3) for each landuse type. When ANOVAs were significant, LSD was used to test differences among means. Tamhane's T2 was used to test differences among treatments when variances of transformed data were not equal. The relationship between recovery rates of nematodes and centrifugation speeds was determined by curve estimation. All statistical analyses used SPSS software (SPSS Inc., Chicago, IL). Statistical significance was determined at $p<0.05$.

\section{Results and discussion}

Within each land-use type, the number of nematodes recovered did not significantly differ between the control and the three methods (Fig. 3). The results indicate that all three methods may be useful for cleaning nematode samples, at least in terms of abundance. Across all land-use types, the mean nematode recovery rate was $94 \%$ for repeated centrifugation (method I), $84 \%$ for the repeated low-temperature settling (method II), and $78 \%$ for the combination of lowtemperature settling and centrifugation (method III) (Supporting information, Fig. S3). An ANOVA indicated that the recovery rate was not significantly greater in using the control method than in using cleaning methods (Supporting information, Fig. S3). In addition, the repeated centrifugation method required less time than the other two cleaning methods. The current results therefore indicate that repeated centrifugation method is the most efficient method for cleaning turbid nematode suspensions.

Lower clarity of the suspensions is required for the counting of nematodes than for their taxonomic analysis (Seinhorst, 1962). In the present study, although taxonomic determinations of the nematodes were not conducted, the purified samples were sufficiently clean for the taxonomic determinations (according to our experience). Moreover, the quantitive analysis was capable of achieving the research goals of this study. Particularly, if the proposed methods significantly reduced the nematode quantity, then the methods are not satisfactory and there is no need for further taxonomic analysis. Since the nematode abundances and recovery rates had no significant differences between the control method and the cleaning methods, the nematode taxonomic compositions are potentially unaffected by the cleaning methods, especially for the repeated centrifugation with the highest nematode recovery rate. Because both quantitative and taxonomic analysis of nematodes are important (Bongers and Ferris, 1999; Neher and Darby, 2009; Ferris and Tuomisto, 2015), additional research may be needed to determine the usefulness of the cleaning methods for taxonomic analysis.

In addition, the procedures of the proposed methods could

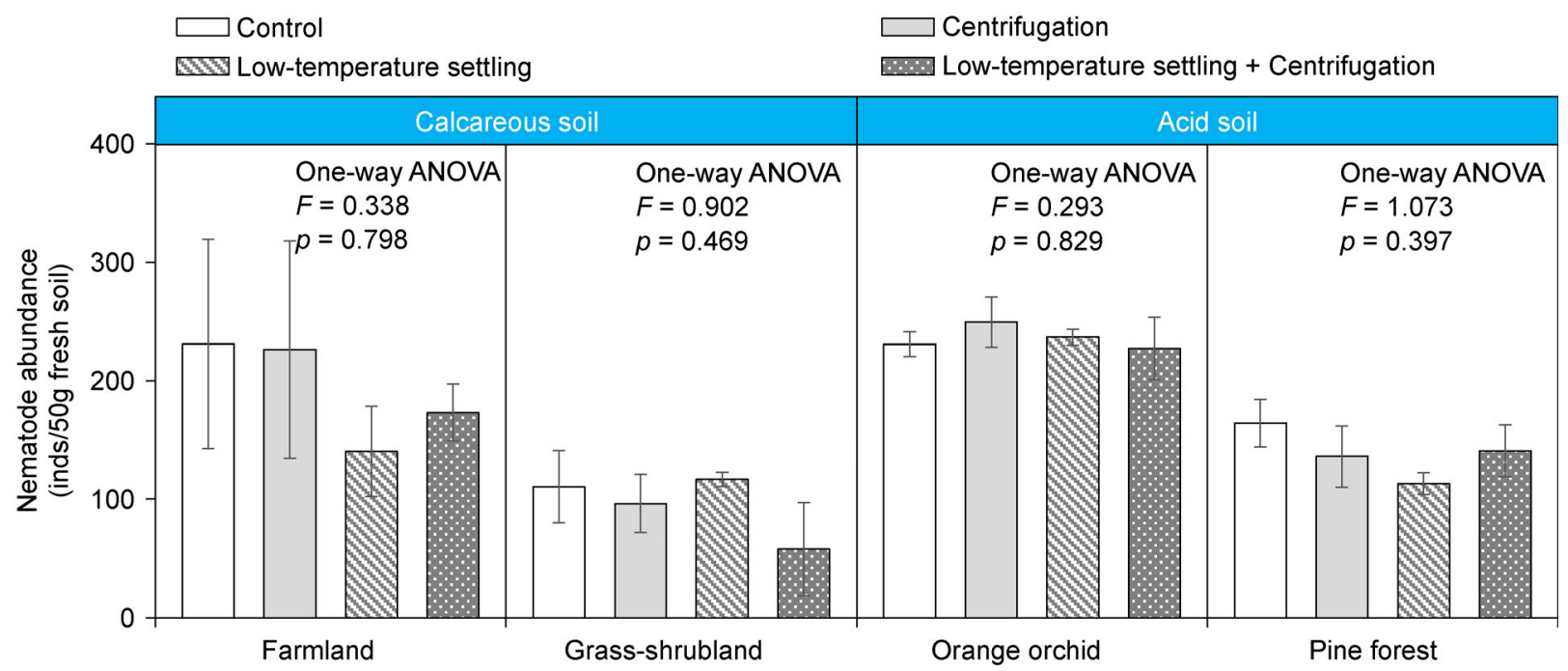

Fig. 3 Numbers of nematodes counted in suspensions obtained from Baermann funnel extraction of soil samples as affected by land-use type and suspension cleaning method. Control: suspension volume was increased to reduce turbidity. Centrifugation: see method I in Fig. 1. Low-temperature settling: see method II in Fig. 1; Low-temperature settling + Centrifugation: see method III in Fig. 1. Values are means \pm SE. 
be changed to further improve their efficiencies. For instance, the repeated centrifugation method was applied to hot formalin fixed samples due to lack of facilities in the field station in this study. According to our experience, the impurities will form flocculent precipitate after hot formalin fixation, which will decrease separation efficiency. Therefore, we suggest using the repeated centrifugation method to purify fresh nematode samples and then fix in hot formalin. In addition, the time for natural low-temperature settling method was $24 \mathrm{~h}$ which may not be necessary. Twelve hours or even shorter time may be enough for the settling of nematodes in $15-\mathrm{ml}$ centrifuge tubes. Moreover, formalin is one of the most commonly used and effective fixatives for nematodes but is toxic (Freckman and Baldwin, 1990; Abebe et al., 2006). Other fixatives with no toxicity may be a choice for certain scenarios. For example, ethanol can be used as a fixative for short-term preservation of nematodes. However, the ethanol often causes specimen distortion (Abebe et al., 2006). Therefore, careful use of formalin in a fume cupboard during fixation and purification of nematodes is recommended.

\section{Acknowledgments}

This study was supported by the Natural Science Fund of China (41877055); Natural Science Foundation for Distinguished Young Scholars of Hunan Province given to Jie Zhao; the Guangxi Natural Science Foundation Program (2018GXNSFAA281008); the Foundation for Young Scholars in Western China of CAS given to Jie Zhao (A class); and the program of the Youth Innovation Promotion Association of Chinese Academy of Sciences (Y201969). The authors have no conflict of interest.

\section{Electronic supplementary material}

Supplementary material is available in the online version of this article at https://doi.org/10.1007/s42832-021-0115-1 and is accessible for authorized users.

\section{References}

Abebe, E., Andrássy, I., Traunspurger, W., 2006. Freshwater nematodes: ecology and taxonomy. CABI.

Aira, M., Domínguez, J., 2020. Soil under dead or live organic matter systems: Effect of European shag (Phalacrocorax aristotelis L.) nesting on soil nematodes and nutrient mineralization. Soil Ecology Letters 2, 40-46.

Barker, K., Carter, C., Sasser, J., 1985. An advanced treatise on Meloidogyne. Volume II: Methodology. United States Agency for International Development, Raleigh, vi +223 pp. pp.

Bongers, T., Ferris, H., 1999. Nematode community structure as a bioindicator in environmental monitoring. Trends in Ecology \& Evolution 14, 224-228.

Chen, X., Xue, W., Xue, J., Griffiths, B.S., Liu, M., 2020. Contribution of bacterivorous nematodes to soil resistance and resilience under copper or heat stress. Soil Ecology Letters 2, 220-229.

Coleman, D., Wall, D., 2015. Soil Fauna: Occurrence, Biodiversity, and Roles in Ecosystem Function, In: Paul, E. (Ed.), Soil Microbiology, Ecology and Biochemistry. Academic, Boston, pp. 111-149.

Coleman, D.C., Blair, J.M., Elliott, E.T., Wall, D.H., 1999. Soil Invertebrates. In: Robertson, G.P., Coleman, D.C., Bledsoe, C. S., Sollins, P. (Eds.), Standard Soil Methods for Long-term Ecological Research. Oxford University Press, New York, pp. 349-377.

Coleman, D.C., Crossley, D.A. Jr, 1996. Fundamentals of soil ecology. Academic Press, London, xii + 205 pp. pp.

FAO, 2006. World reference base for soil resources 2006. World Soil Resources Reports No. 103. FAO, Rome.

Ferris, H., Tuomisto, H., 2015. Unearthing the role of biological diversity in soil health. Soil Biology \& Biochemistry 85, 101-109.

Freckman, D., Baldwin, J., 1990. Nematoda, In: Dindal, D.L. (Ed.), Soil Biology Guide. Wiley, New York, pp. 155-200.

Geisen, S., Snoek, L.B., ten Hooven, F.C., Duyts, H., Kostenko, O., Bloem, J., Martens, H., Quist, C.W., Helder, J.A., der Putten, W.H., 2018. Integrating quantitative morphological and qualitative molecular methods to analyse soil nematode community responses to plant range expansion. Methods in Ecology and Evolution 9, 1366-1378.

Huang, F., Wei, H., Cao, J., 2018. Response characteristics of soil microbes on maize straw amendment-A typical comparison between subtropical limestone soil and red soil. Journal of Southern Agriculture, 49.

Li, J., Peng, P., Zhao, J., 2020. Assessment of soil nematode diversity based on different taxonomic levels and functional groups. Soil Ecology Letters 2, 33-39.

Neher, D.A., 2001. Role of nematodes in soil health and their use as indicators. Journal of Nematology 33, 161-168.

Neher, D.A., Darby, B.J., 2009. General community indices that can be used for analysis of nematode assemblages, In: Wilson, M.J., Kakouli-Duarte, T. (Eds.), Nematodes as Environmental Indicators. CABI, Wallingford, pp. 107-123.

Sarathchandra, S.U., Ghani, A., Yeates, G.W., Burch, G., Cox, N.R., 2001. Effect of nitrogen and phosphate fertilisers on microbial and nematode diversity in pasture soils. Soil Biology \& Biochemistry 33, 953-964.

Seinhorst, J.W., 1962. Modifications of the clutriation method for extracting nematodes from soil. Nematologica 8, 117-128.

Wright, C.J., Coleman, D.C., 2000. Cross-site comparison of soil microbial biomass, soil nutrient status, and nematode trophic groups. Pedobiologia 44, 2-23.

Xiao, M., Chen, X., Li, Y., He, X., Shen, Y., Su, Y., 2014. Carbon release from brown limestone and red soils in response to addition of $\mathrm{Fe}(\mathrm{OH})_{3}$ and $\mathrm{CaCO}_{3}$. Chinese Journal of Ecology 33, 29362942 (in Chinese with English abstract).

Zhang, X., Liang, W., Qi, L., 2013. Forest Soil Nematodes in Changbai Mountain-Morphology and Distribution. China Agricultural Press, Beijing.

Zhang, X., Wu, X., Zhang, S., Xing, Y., Liang, W., 2019. Organic amendment effects on nematode distribution within aggregate 
fractions in agricultural soils. Soil Ecology Letters 1, 147-156.

Zhao, J., Wan, S., Li, Z., Shao, Y., Xu, G., Liu, Z., Zhou, L., Fu, S., 2012. Dicranopteris-dominated understory as major driver of intensive forest ecosystem in humid subtropical and tropical region. Soil Biology \& Biochemistry 49, 78-87.

Zhao, J., Xun, R., He, X., Zhang, W., Fu, W., Wang, K., 2015. Size spectra of soil nematode assemblages under different land use types. Soil Biology \& Biochemistry 85, 130-136. 